\section{Association of antibiotic treatment with immune-related adverse events in patients with cancer receiving immunotherapy}

To cite: Jing Y, Chen X, Li K, et al. Association of antibiotic treatment with immune-related adverse events in patients with cancer receiving immunotherapy. Journal for ImmunoTherapy of Cancer 2022;10:e003779. doi:10.1136/ jitc-2021-003779

- Additional supplemental material is published online only. To view, please visit the journal online (http://dx.doi.org/10. 1136/jitc-2021-003779).

YJ and XC contributed equally. Accepted 11 December 2021

Check for updates

(C) Author(s) (or their employer(s)) 2022. Re-use permitted under CC BY-NC. No commercial re-use. See rights and permissions. Published by BMJ.

For numbered affiliations see end of article.

\section{Correspondence to \\ Dr Leng Han;}

leng.han@tamu.edu

Dr Hong Liu; hongliu1014@csu.edu.cn

Dr Xiang Chen; chenxiangck@126.com

\section{ABSTRACT}

Background To determine whether antibiotic treatment is a risk factor for immune-related adverse events (irAEs) across different patients with cancer receiving anti-PD-1/ PD-L1 therapies.

Methods The retrospective analysis includes clinical information from 767 patients with cancer treated at Hunan Cancer Hospital from 2017 to 2020 . The pharmacovigilance data analysis includes individual cases of 38,705 safety reports from the US Food and Drug Administration Adverse Event Reporting System (FAERS) from 2014 to 2020, and 25,122 cases of safety reports from the World Health Organization database VigiBase from 2014 to 2019. All cases that received anti-PD-1/PDL1 treatment were included. Multiomics data from patients across 25 cancer types were download from The Cancer Genome Atlas. Logistic regression and propensity score algorithm was employed to calculate OR of irAEs. Results Retrospective analysis of in-house patients showed that irAE potential risks are higher in all cancer (OR 2.12, 95\% $\mathrm{Cl} 1.38$ to 3.22, false discovery rate (FDR) adjusted- $p=1.93 \times 10^{-3}$ ) and patients with lung cancer (OR $3.16,95 \% \mathrm{Cl} 1.67$ to 5.95 , FDR adjusted- $p=1.93 \times 10^{-3}$ ) when using antibiotics. Potential risk of irAEs in patients with lung cancer with antibiotic treatment is significantly higher in FAERS $(\mathrm{OR} 1.39,95 \% \mathrm{Cl} 1.21$ to 1.59 ; FDR adjusted- $\left.p=1.62 \times 10^{-5}\right)$ and VigiBase (OR $1.32,95 \% \mathrm{Cl}$ 1.09 to 1.59 , FDR adjusted- $p=0.05$ ). Mechanistically, decreased microbial diversity caused by antibiotics use may increase the irAE risk through mediating the irAErelated factors.

Conclusions Our study is the first to comprehensively demonstrate the associations of irAEs and antibiotic during anti-PD-1/PD-L1 therapy across a wide spectrum of cancers by analyzing multisource data. Administration of antibiotics should be carefully evaluated in patients with cancer treated by anti-PD-1/PD-L1 to avoid potentially increasing irAE risk.

\section{BACKGROUND}

Immune checkpoint inhibitors (ICIs) have shown striking benefit in a wide spectrum of cancer types, but may also lead to a series of immune-related adverse events (irAEs) that may affect any organ. ${ }^{1-3}$ Severe and fatal irAEs have been reported, ${ }^{4-6}$ such as myocarditis, ${ }^{56}$ pneumonitis, ${ }^{4}$ and colitis. ${ }^{4}$ These immune-related toxicities limit the benefits of ICIs and could lead to discontinuation of ICIs. ${ }^{78}$ A comprehensive understanding of irAEs induced by immunotherapy is important for managing the benefit/risk ratio of immunotherapy. ${ }^{9}$ Exposure to antibiotics has been associated with less clinical benefit from ICIs in lung cancer, ${ }^{10}{ }^{11}$ melanoma ${ }^{11}$ and renal cell carcinoma. ${ }^{10}$ However, there is minimal evidence about the association between irAE development and antibiotic use in ICI patients. ${ }^{12} 13$ Previous studies reported that antibiotic use is associated with higher risk of diarrhea and colitis, which are among gastrointestinal irAEs, in ICI patients. ${ }^{12} 13$ However, it is not clear whether antibiotic use impacts irAEs in organs other than the gastrointestinal tract during ICI treatment. Therefore, a comprehensive, in-depth characterization of the association of irAEs with antibiotic treatment is crucial.

In this study, we retrospectively analyzed clinical information of ICI patients within an in-house patient cohort and observed increased risk of irAEs in lung cancer ICI patients who were given antibiotics. To obtain a more generalized conclusion, we took advantage of large-scale pharmacovigilance data from the US Food and Drug Administration Adverse Event Reporting System (FAERS) and the WHO pharmacovigilance database (VigiBase) and observed similar pattern for specific irAEs. Recent studies demonstrated the impact of human microbiomes on cancer progression and therapy, ${ }^{14-16}$ so we further obtained the microbial data from The Cancer Genome Atlas (TCGA) to understand the potential underlie mechanism through multiomics data. Taken together, our study employed multisource 
evidence aiming to investigate the administration of antibiotics and increasing irAE risk in patients with cancer treated by anti-PD-1/PD-L1.

\section{METHODS}

\section{Retrospective analysis of in-house patients}

We performed a retrospective analysis of 767 patients with cancer treated with anti-PD-1/anti-PD-L1 at Hunan Cancer Hospital,the Affiliated Cancer Hospital of Xiangya School of Medicine between 2017 and 2020. Clinical information, including age at diagnosis, sex, cancer types, response, clinical interventions, duration of ICI treatment, history of antibiotic treatment, and ICI drug and combinations, was obtained from the medical records. Data regarding irAEs were collected, including type, symptoms, and grade of irAEs (according to common terminology criteria for adverse events, V.4.0). Patients who received antibiotics within 3 months before or after the first ICI administration were identified as antibiotic users according to previous studies. ${ }^{12} 1317$ Specifically, patients who received antibiotics within 3 months before the first dose of ICIs were classified as pre-ICI group. Multivariable logistic regression models were employed to calculate the adjusted OR. ${ }^{18-20}$ The variables analyzed in the model were age, sex, ICI drugs and combination therapy.

\section{Pharmacovigilance data analysis}

Individual adverse event (AE) reports between July 1, 2014 and June 30, 2020 were downloaded from the FAERS website (https://www.fda.gov/drugs/questionsand-answers-fdas-adverse-event-reporting-system-faers / fda-adverse-event-reporting-system-faers-public-dashboard) and reports between July 1, 2014 and December 31, 2019 were queried from VigiBase (https://www.whoumc.org/vigibase/vigibase/). We collected AE reports from anti-PD-1 agents (nivolumab, pembrolizumab, cemiplimab) and anti-PD-L1 agents (atezolizumab, avelumab, durvalumab) suspected of causing AEs across different cancer types, as previously described. ${ }^{21}$ Patients who received antibiotics during the anti-PD-1/PD-L1 therapies were identified as antibiotic users. Patients with only one clearly defined cancer type were included. Patients of ages 0-100 years were included. Cases for which the patient's sex was not reported were excluded. Adjusted ORs of irAEs based on FAERS data were analyzed by multivariable logistic regression. ${ }^{18-20}$ The variables analyzed in the model were age, sex, antibiotic use, and different ICI drugs. Considering that pharmacovigilance data records does not allow to obtain results on 'risk' to individual patients due to the intrinsic limitations of the database, we only obtain results on potential risk through calculating OR.

\section{Determination of irAEs in pharmacovigilance data}

We used the $\mathrm{AE}$ terms in peer-reviewed management guidelines $^{22}$ to define irAEs, including hepatitis, StevensJohnson syndrome, and colitis (online supplemental table S1) to determine patients with irAEs. This prespecified list is one of the most comprehensive and accurate irAE list so far, at least under the immunotherapy settings. Patients were classified into the irAE group if they had at least one irAE from this guideline. The irAEs were grouped into primary system organ classes based on the Medical Dictionary for Drug Regulatory Activities, V.23.0.

\section{Microbial diversity and molecular data from TCGA}

Microbial reads data of TCGA samples were obtained from a previous study. ${ }^{23}$ Inverse Simpson index were calculated from all microbial reads in each sample via 'diversity' function in $R$ package 'vegan'. We analyzed omics data from TCGA for 25 cancer types with $\geq 100$ samples. The irAE information is lacking in TCGA for individual patients, so we investigate the associations between microbial diversity and previously reported irAE-related factors and signatures: tumor mutational burden (TMB), ${ }^{24} \mathrm{~T}$ cell receptor (TCR) diversity, ${ }^{25}$ neutrophils, ${ }^{26}$ eosinophils, ${ }^{26}$ CEACAM $1,{ }^{26} \mathrm{CD} 177,{ }^{26}$ interferon (IFN) alpha response, ${ }^{27}$ tumor necrosis factor (TNF) signature, ${ }^{27}$ cytolytic activity, ${ }^{21} \mathrm{ADPGK},{ }^{21} \mathrm{LCP} 1,{ }^{21} \mathrm{PD}-1,{ }^{21}$ and potential pathway ${ }^{28}$ related to $\mathrm{T}$ cell activation, neutrophil activation, eosinophils, and inflammation. Molecular data, including mRNA expression and mutations, were downloaded from TCGA data portal (https://portal.gdc.cancer. gov/). T-cell receptor diversity, and estimated immune cell abundance were downloaded from the Genomic Data Commons (GDC) PanImmune Data Portal (https://gdc. cancer.gov/about-data/publications/panimmune). ${ }^{29}$ TMB was calculated by the number of non-silent somatic mutations per sample. ${ }^{30}$ Pathways related to T-cell activation, neutrophil activation, eosinophil activation and inflammation, and IFN $\alpha$ response were obtained from MSigDB (http:// software.broadinstitute.org/gsea/msigdb/genesets.jsp); TNF signatures were obtained from Perez-Ruiz et al. ${ }^{31}$ IFN $\gamma$ signature was obtained from Ayers et al. ${ }^{32}$ TNF signature and IFN $\gamma$ signature were calculated using the GSVA ${ }^{33} R$ package. Spearman correlation were performed to analyze the associations between microbial diversity and omics data. Pathway enrichment was conducted using the $\mathrm{R}$ package 'clusterprofiler' ${ }^{34}$ Genes with absolute Rs value $\geq 0.3$ and false discovery rate (FDR)-adjusted $\mathrm{p}<0.05$, obtained from spearman correlation, were used in pathway enrichment.

\section{Statistical analysis}

Multiple comparisons were Benjamini-Hochberg adjusted by passing the test $\mathrm{p}$ values to the 'p.adjust' function of the 'stats' $R$ package. All reported $p$ values are two sided; FDR-adjusted $\mathrm{p}<0.1$ and $\mathrm{p}<0.05$ were considered significant. Data processing and statistical analyses were performed using R statistical software V.3.5.1.

\section{RESULTS}

Retrospective analysis identified positive association between antibiotic use and irAEs in lung cancer

We conducted retrospective analysis of 767 patients with cancer receiving anti-PD-1/PD-L1 therapy in an in-house patient cohort (figure 1A). Among these patients, 340 
A

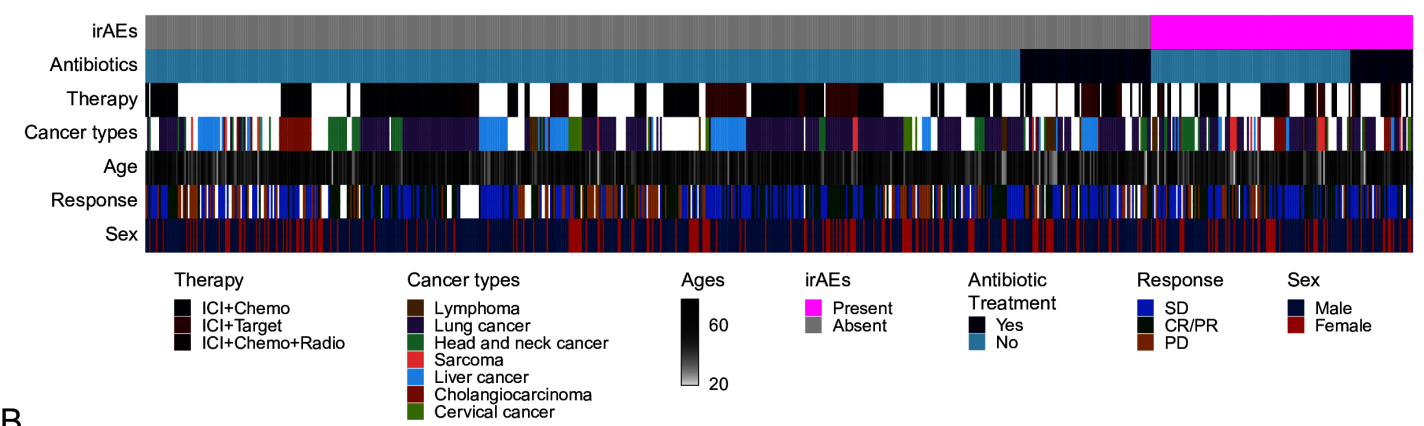

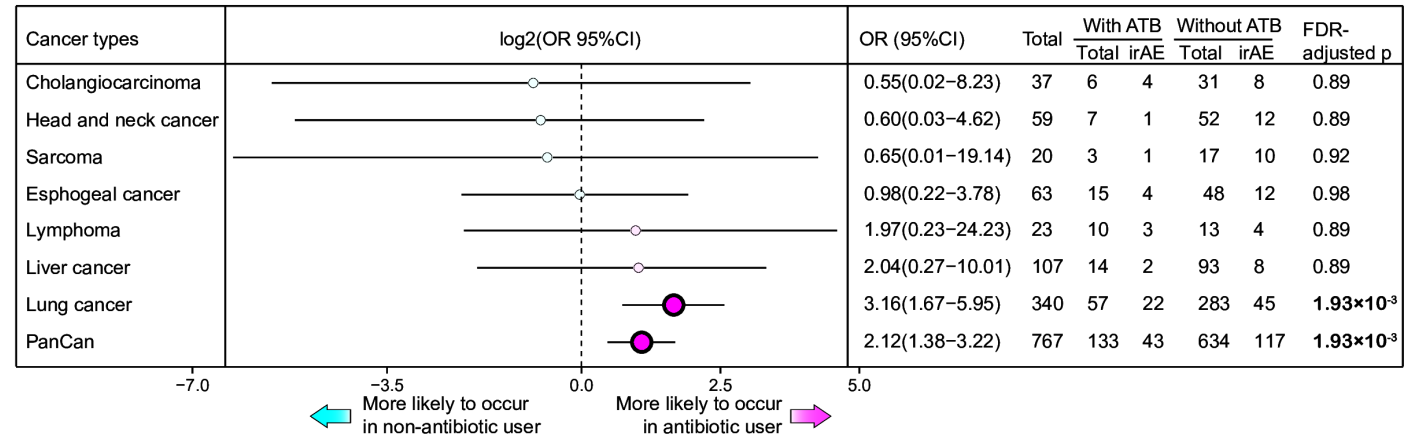

\begin{tabular}{|c|c|c|c|c|c|c|c|}
\hline Time & $\log 2(\mathrm{OR} 95 \% \mathrm{Cl})$ & OR $(95 \% \mathrm{Cl})$ & Total & $\frac{\text { With ATB }}{\text { Total irAE }}$ & $\frac{\text { Witho }}{\text { Total }}$ & $\frac{\text { at ATB }}{\text { irAE }}$ & $p$-value \\
\hline pre-ICl & $-c$ & $2.87(1.08-7.23)$ & 655 & 21 & 634 & 110 & 0.03 \\
\hline
\end{tabular}

$\prec \begin{aligned} & \text { More likely to occur } \\ & \text { in non-antibiotic user }\end{aligned}$
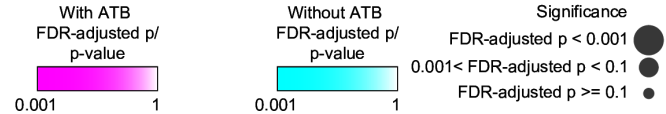

Figure 1 Retrospective analysis for in-house patient cohorts. (A) Clinical features of in-house anti-PD-1/PD-L1 cancer patient cohort. Each row represents an individual patient. (B) Analysis of immune-related adverse events (irAEs) in antibiotic and non-antibiotic users among patients with different cancer types receiving anti-PD-1/PD-L1. (C) Difference in association between antibiotics administered 3 months before (pre-ICl) first dose of $\mathrm{ICl}$ therapies and irAE risks in all patients with cancer. Magenta indicates that irAEs are more likely to occur in antibiotic users; cyan indicates that irAEs are more likely to occur in non-antibiotic users; shade of the dot indicates false discovery rate (FDR)-adjusted $p$ value/ $p$-value. Dot size from large to small respectively indicates FDR-adjusted $p / p$-value $<0.001,0.001<F D R$-adjusted $p / p$-value $<0.1$, and FDR-adjusted $p / p$-value $\geq 0.1$, as shown. ATB, antibiotics; CR, complete response; ICl, immune checkpoint inhibitor; irAE, immune-related adverse events; PR, partial response; PD, progressive disease; SD, stable disease.

were patients with lung cancer (44.3\%, table 1$)$ and 107 were patients with liver cancer $(14.0 \%$, table 1$)$. Patients who received antibiotics treatment were classified as antibiotic users. We used multivariable logistic regression analysis, adjusting for age, sex, ICI drug, and combination therapy to identify the association between antibiotic treatment and irAEs. We observed that antibiotic use increased irAE risk in all patients with cancer (adjusted OR $2.12,95 \%$ CI 1.38 to 3.22 , FDR-adjusted $p=1.93 \times 10^{-3}$, figure 1B). However, this significant association was likely driven by patients with lung cancer (OR 3.16, 95\% CI 1.67 to 5.95 , FDR-adjusted $\mathrm{p}=1.93 \times 10^{-3}$, figure $1 \mathrm{~B}$ ), who represented the largest sample size. We did not observe significant associations in other cancer types (figure 1B). This may be due to the limited sample size in other cancer types, and further investigations were necessary to obtain more robust results. Considering that some patients with cancer will be given antibiotics for irAE management, ${ }^{22}$ we explored the possibility of an association between antibiotics administered within 3 months ${ }^{121317}$ before first dose of immune checkpoint inhibitor (ICI) therapy and irAEs (pre-ICI). We observed similarly significant increased irAE risk in pre-ICI group (OR 2.87, 95\% CI 1.08 to 7.23; $\mathrm{p}=0.03$; figure $1 \mathrm{C}$ ) as in patients received antibiotics at any time (figure 1B). Additional analysis on patients received antibiotics within 30 days ${ }^{115-38}$ before the first dose of ICIs was performed (pre-ICI 30-days, OR 3.66, 95\% CI 1.23 to 10.52; $\mathrm{p}=0.02$; online supplemental figure $\mathrm{S} 1$ ). These increased risks in both pre-ICI groups with antibiotics usage suggests that the association between irAEs and antibiotic usage is unlikely to be caused by concurrent antibiotics during irAE management. Moreover, 
Table 1 Characteristics of 767 patients from in-house cohort

\begin{tabular}{|c|c|c|}
\hline & Antibiotic user & Non-antibiotic user \\
\hline Characteristic & $(n=133)$ & $(n=634)$ \\
\hline \multicolumn{3}{|l|}{ Sex, no (\%) } \\
\hline Male & $94(70.7)$ & $472(74.4)$ \\
\hline Female & 39 (29.3) & $162(25.6)$ \\
\hline Median (IQR) age, years & $55(47-65)$ & $55(49-63)$ \\
\hline \multicolumn{3}{|l|}{ Cancer type, no (\%) } \\
\hline Lung cancer & $57(42.9)$ & $283(44.6)$ \\
\hline Liver cancer & $14(10.5)$ & $93(14.7)$ \\
\hline Esophageal cancer & $15(11.3)$ & $48(7.6)$ \\
\hline Head and neck cancer & $7(5.3)$ & $52(8.2)$ \\
\hline Cholangiocarcinoma & $6(4.5)$ & $31(4.9)$ \\
\hline Cervical cancer & $10(7.5)$ & $20(3.2)$ \\
\hline Lymphoma & $10(7.5)$ & $13(2.1)$ \\
\hline Sarcoma & $3(2.3)$ & $17(2.7)$ \\
\hline Other & $11(8.3)$ & $77(12.1)$ \\
\hline \multicolumn{3}{|l|}{ irAEs, no (\%) } \\
\hline Present & $43(32.3)$ & $110(17.4)$ \\
\hline Absent & $90(67.7)$ & $524(82.6)$ \\
\hline \multicolumn{3}{|l|}{ Drugs, no (\%) } \\
\hline anti-PD-1 & $98(73.7)$ & $474(74.8)$ \\
\hline anti-PD-L1 & $35(26.3)$ & $160(25.2)$ \\
\hline \multicolumn{3}{|l|}{ Therapy, no (\%) } \\
\hline $\mathrm{ICI}$ only & 69 (51.9) & $282(44.5)$ \\
\hline $\mathrm{ICI}+$ chemotherapy & $42(31.6)$ & $261(41.2)$ \\
\hline $\mathrm{ICI}+$ target therapy & $22(16.5)$ & $77(12.1)$ \\
\hline $\mathrm{ICl}+$ chemo+radiotherapy & $0(0)$ & $14(2.2)$ \\
\hline \multicolumn{3}{|l|}{ Grade of irAEs, no (\%) } \\
\hline Grade 1 & $24(55.81)$ & $59(50.43)$ \\
\hline Grade 2 & $9(20.93)$ & $33(28.21)$ \\
\hline Grade 3 & $4(9.30)$ & $11(9.40)$ \\
\hline Grade 4 & $1(2.33)$ & $0(0.00)$ \\
\hline Grade 5 & $1(2.33)$ & $1(0.85)$ \\
\hline Unknown & $4(9.30)$ & $13(11.11)$ \\
\hline \multicolumn{3}{|l|}{ Specified irAEs, no (\%) } \\
\hline Thyroid irAEs & $16(27.12)$ & $41(29.71)$ \\
\hline Vascular irAEs & $6(10.17)$ & $15(10.87)$ \\
\hline Rash & $5(8.47)$ & $14(10.14)$ \\
\hline Hepatic irAEs & $5(8.47)$ & $13(9.42)$ \\
\hline Hematologic irAEs & $2(3.39)$ & $11(7.97)$ \\
\hline Gastrointestinal irAEs & $4(6.78)$ & $10(7.25)$ \\
\hline Others & $5(8.47)$ & $8(5.80)$ \\
\hline Pneumonitis & $7(11.86)$ & $7(5.07)$ \\
\hline Renal irAEs & $1(1.69)$ & $4(2.90)$ \\
\hline Skin irAEs & $4(6.78)$ & $3(2.17)$ \\
\hline Cardiac irAEs & $2(3.39)$ & $3(2.17)$ \\
\hline
\end{tabular}


Table 1 Continued

\begin{tabular}{|c|c|c|}
\hline & Antibiotic user & Non-antibiotic user \\
\hline Characteristic & $(n=133)$ & $(n=634)$ \\
\hline Endocrine irAEs & $0(0.00)$ & $3(2.17)$ \\
\hline Eye irAEs & $1(1.69)$ & $2(1.45)$ \\
\hline Musculoskeletal irAEs & $0(0.00)$ & $1(0.72)$ \\
\hline \multicolumn{3}{|l|}{ Duration of treatment } \\
\hline$\leq 1$ month & $9(6.77)$ & $30(4.73)$ \\
\hline 1-3 months & $33(24.81)$ & $119(18.77)$ \\
\hline 3-6 months & $28(21.05)$ & $127(20.03)$ \\
\hline
\end{tabular}

$\mathrm{ICl}$, immune checkpoint inhibitor; irAEs, immune-related adverse events.

considering patients who received benefit from ICIs may have longer treatment period and higher chance to develop irAEs, ${ }^{39}{ }^{40}$ we further added the duration of treatment as covariates in logistic regression model, and observed a similar pattern (online supplemental figure S2). Next, we performed subgroup analysis for specific irAEs, which shown that risks of pneumonitis (OR $6.49,95 \%$ CI 2.05 to 20.53 , FDR-adjusted $\mathrm{p}=1.03 \times 10^{-2}$ ) and thyroid irAEs (OR 2.18, 95\% CI 1.16 to 4.10 , FDRadjusted $\mathrm{p}=0.05$ ) were higher in antibiotic users, and we also observed similar trend for most of the rest irAE types (online supplemental figure S3A). Moreover, grade 1-2 (OR 2.04, 95\% CI 1.28 to 3.24 , FDR-adjusted $\mathrm{p}=5.16 \times 10^{-3}$ ) and grade $3-5$ irAEs (OR 2.80, 95\% CI 1.02 to 7.73, FDRadjusted $\mathrm{p}=4.65 \times 10^{-2}$ ) were both significantly associated with antibiotics usage (online supplemental figure S3B).

\section{Association between antibiotic use and irAEs in FAERS and VigiBase}

Despite the relatively large sample size of our in-house patient cohort, it was not comparable to the size of the real-world pharmacovigilance database. ${ }^{2141} \mathrm{We}$, therefore, interrogated two global pharmacovigilance databases, FAERS and VigiBase, to explore associations between antibiotic use and irAEs in a more comprehensive manner. FAERS and VigiBase contain hundreds of thousands of patients with cancer receiving immunotherapy. Due to the intrinsic limitations of the database, ${ }^{1735}$ including incompleteness of clinical information and duplicated reports, we can only obtain potential risk through calculating OR as previous studies. ${ }^{18} 19$ We downloaded safety reports of 38,705 patients receiving anti-PD-1/PD-L1 from FAERS (online supplemental figure S2). Of these patients, 18,321 $(47.3 \%)$ were patients with lung cancer and 5688 (14.7\%) were melanoma patients (online supplemental figure S2). A total of $2628(6.8 \%)$ ICI patients were identified as antibiotic users. In the multivariable logistic regression analysis, after adjusting for age, sex and ICI drug, the use of antibiotics was associated with higher irAE risk in pan-caner analysis (OR 1.22, 95\% CI 1.11 to 1.34 ; FDRadjusted $\mathrm{p}=2.87 \times 10^{-4}$; figure $2 \mathrm{~A}$ ). We included 16 cancer types with sample sizes larger than 100 in the analysis for each cancer. Among these cancer types, the use of antibiotics was associated with higher irAE risk in patients with lung cancer (OR 1.39, 95\% CI 1.21 to 1.59; FDR-adjusted $\mathrm{p}=1.62 \times 10^{-5}$; figure $2 \mathrm{~A}$ ) and patients with pancreatic cancer (OR 4.61, 95\% CI 2.56 to 8.32; FDR-adjusted $\mathrm{p}=6.09 \times 10^{-6}$; figure $2 \mathrm{~A}$ ). To further validate our observation, we retrieved safety reports of 25,122 cases receiving anti-PD-1/PD-L1 therapies from VigiBase. Of these patients, 13,594 (54.1\%) were patients with lung cancer, and $4244(16.9 \%)$ were melanoma patients. Antibiotics had been used to treat $1158(4.6 \%)$ of 25,122 patients (online supplemental figure S3). We also observed an association between antibiotic use and increased irAE risk in patients with lung cancer (OR 1.32, 95\% CI 1.09 to 1.59 , FDR-adjusted $\mathrm{p}=0.05$, figure $2 \mathrm{~B}$ ). Taken together, the analyses based on FAERS and VigiBase consistently pinpointed that antibiotic users had higher irAE risk when compared with non-antibiotic users, at least for patients with lung cancer, which is consistent with the findings from our retrospective analysis of the in-house patient cohort.

\section{Association between antibiotic use and irAEs in different organs}

To investigate the association of organ-specific irAEs and use of antibiotics in lung cancer, we grouped the irAEs into 12 organs/systems and performed subgroup analysis. In patients with lung cancer, the risk of renal and urinary irAEs (OR 2.54, 95\% CI 1.49 to 4.09, FDRadjusted $\mathrm{p}=1.09 \times 10^{-3}$ ), hepatobiliary irAEs (OR 2.05, 
A

FAERS

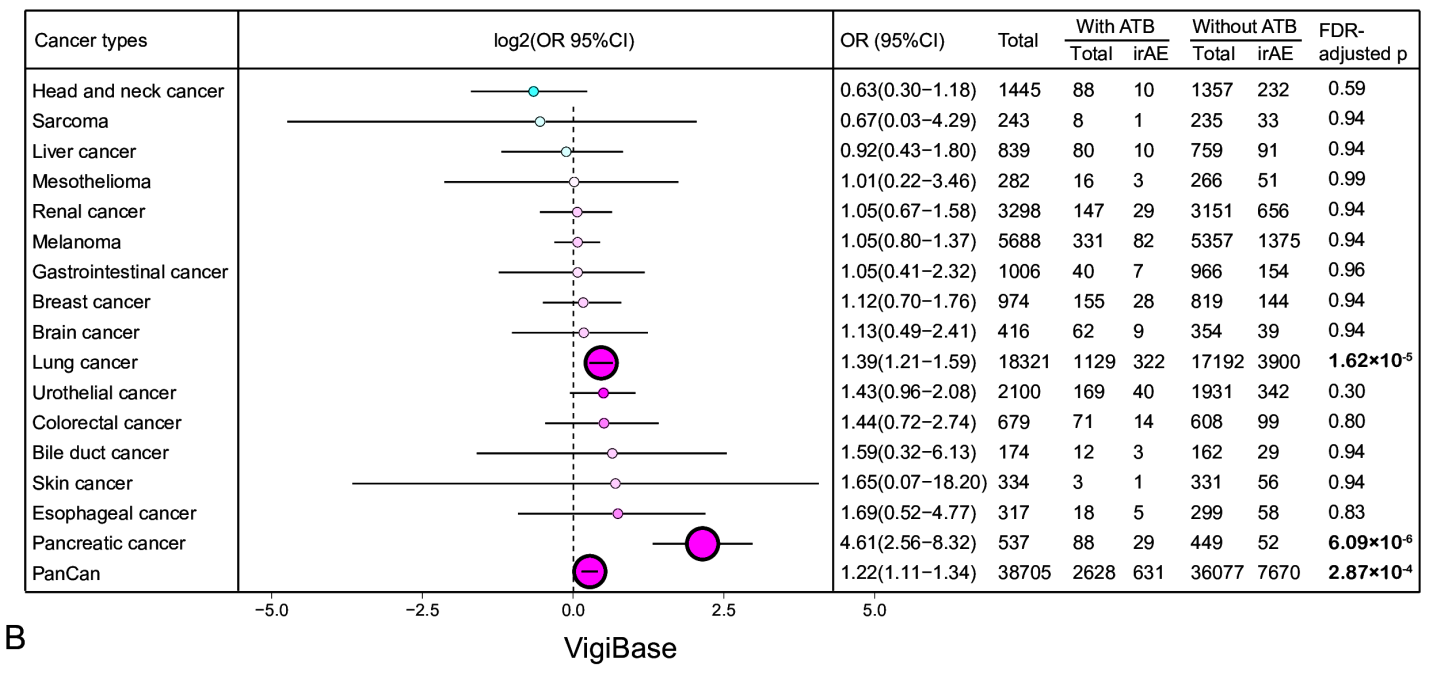

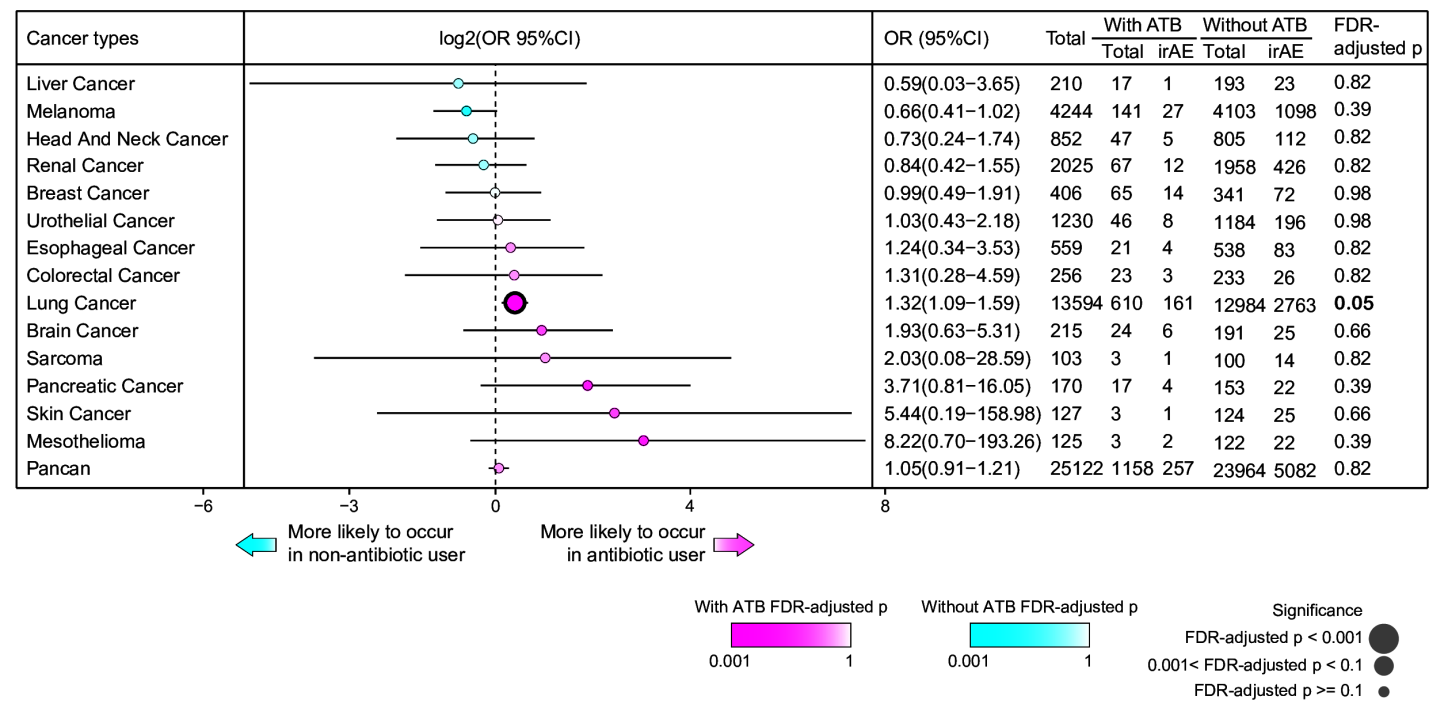

Figure 2 Multivariable logistic regression analysis on pharmacovigilance data for association between antibiotic use and irAEs across different cancer types. Analysis of irAEs in antibiotic and non-antibiotic users among patients with different cancer types receiving anti-PD-1/PD-L1 from Food and Drug Administration Adverse Event Reporting System (FAERS) (A) and VigiBase (B). Magenta indicates that irAEs are more likely to occur in antibiotic users; cyan indicates that irAEs are more likely to occur in non-antibiotic users; shade of the dot indicates false discovery rate (FDR)-adjusted $p$ value. Dot size from large to small respectively indicates FDR-adjusted $p<0.001,0.001<$ FDR-adjusted $p<0.1$, and FDR-adjusted $p \geq 0.1$. ATB, antibiotics.

95\% CI 1.12 to 3.47, FDR-adjusted $\mathrm{p}=0.04$ ), blood and lymphatic system irAEs (OR $1.90,95 \%$ CI 1.51 to 2.36, FDR-adjusted $\left.\mathrm{p}=1.52 \times 10^{-7}\right)$, and respiratory, thoracic and mediastinal irAEs (OR 1.69, 95\% CI 1.31 to 2.15 , FDRadjusted $\mathrm{p}=1.76 \times 10^{-4}$ ) were significantly higher among antibiotic users from the FAERS database (figure 3A). From VigiBase data, we only observed increased risk of respiratory, thoracic and mediastinal irAEs (OR 1.56, $95 \% \mathrm{CI} 1.15$ to 2.09 , FDR-adjusted $\mathrm{p}=0.02$ ) and blood and lymphatic system irAEs (OR 3.15, 95\% CI 2.21 to 4.36, FDR-adjusted $\left.\mathrm{p}=3.67 \times 10^{-10}\right)$ among antibiotic users (figure 3B). Most of the irAEs belonging to respiratory, thoracic and mediastinal disorders were pneumonitis (online supplemental figure S4), which also has been reported to be more common among patients with lung cancer administered anti-PD-1/PD-L1 therapies in clinical trials. ${ }^{122}$
Hence, we further analyzed pneumonitis and other specific irAEs among antibiotic user and nonuser groups and discovered that the development of pneumonitis was also significantly associated with the use of antibiotics in patients with lung cancer receiving anti-PD-1/PD-L1 in FAERS (OR 1.71, 95\% CI 1.32 to 2.18; FDR-adjusted $\mathrm{p}=4.61 \times 10^{-4}$, figure $4 \mathrm{~A}$ ) and VigiBase (OR $1.56,95 \% \mathrm{CI}$ 1.14 to 2.09 ; FDR-adjusted $p=0.04$; figure $4 B$ ). Similarly, the risk of thrombocytopenia, anemia and neutropenia (the blood and lymphatic system) in patients with lung cancer who used antibiotics was also higher (figure 4A-B). These results suggested that the administration of antibiotics to patients with lung cancer with anti-PD-1/PD-L1 therapy increased the risk of irAEs, especially for immune-related pneumonitis. 
A

FAERS lung cancer

\begin{tabular}{|c|c|c|c|c|c|c|c|c|}
\hline \multirow{2}{*}{ irAE SOC } & \multirow{2}{*}{$\log 2(\mathrm{OR} 95 \% \mathrm{Cl})$} & \multirow{2}{*}{ OR $(95 \% \mathrm{Cl})$} & \multirow{2}{*}{ Total } & \multicolumn{4}{|c|}{ With ATB Without ATB } & \multirow{2}{*}{$\begin{array}{l}\text { FDR- } \\
\text { adjusted } p\end{array}$} \\
\hline & & & & Total & $\overline{\operatorname{irAE}}$ & Total & irAE & \\
\hline Endocrine & & $0.50(0.21-0.99)$ & 18321 & 1129 & 7 & 17192 & 210 & 0.18 \\
\hline Vascular & & $0.61(0.03-2.88)$ & 18321 & 1129 & 1 & 17192 & 25 & 0.69 \\
\hline Musculoskeletal and connective tissue & & $0.73(0.48-1.06)$ & 18321 & 1129 & 26 & 17192 & 543 & 0.23 \\
\hline Skin and subcutaneous tissue & & $0.89(0.66-1.17)$ & 18321 & 1129 & 53 & 17192 & 908 & 0.57 \\
\hline Eye & & $1.09(0.33-2.67)$ & 18321 & 1129 & 4 & 17192 & 55 & 0.86 \\
\hline Cardiac & & $1.15(0.67-1.82)$ & 18321 & 1129 & 17 & 17192 & 227 & 0.69 \\
\hline Gastrointestinal & & $1.20(0.84-1.66)$ & 18321 & 1129 & 37 & 17192 & 472 & 0.45 \\
\hline Nervous system & & $1.38(0.81-2.20)$ & 18321 & 1129 & 17 & 17192 & 189 & 0.36 \\
\hline Respiratory, thoracic and mediastinal & & $1.69(1.31-2.15)$ & 18321 & 1129 & 75 & 17192 & 692 & $1.76 \times 10^{-4}$ \\
\hline Blood and lymphatic system & & $1.90(1.51-2.36)$ & 18321 & 1129 & 96 & 17192 & 797 & $1.52 \times 10^{-7}$ \\
\hline Hepatobiliary & & $2.05(1.12-3.47)$ & 18321 & 1129 & 14 & 17192 & 105 & 0.04 \\
\hline Renal and urinary & & $2.54(1.49-4.09)$ & 18321 & 1129 & 18 & 17192 & 109 & $1.09 \times 10^{-3}$ \\
\hline
\end{tabular}

B

VigiBase lung cancer

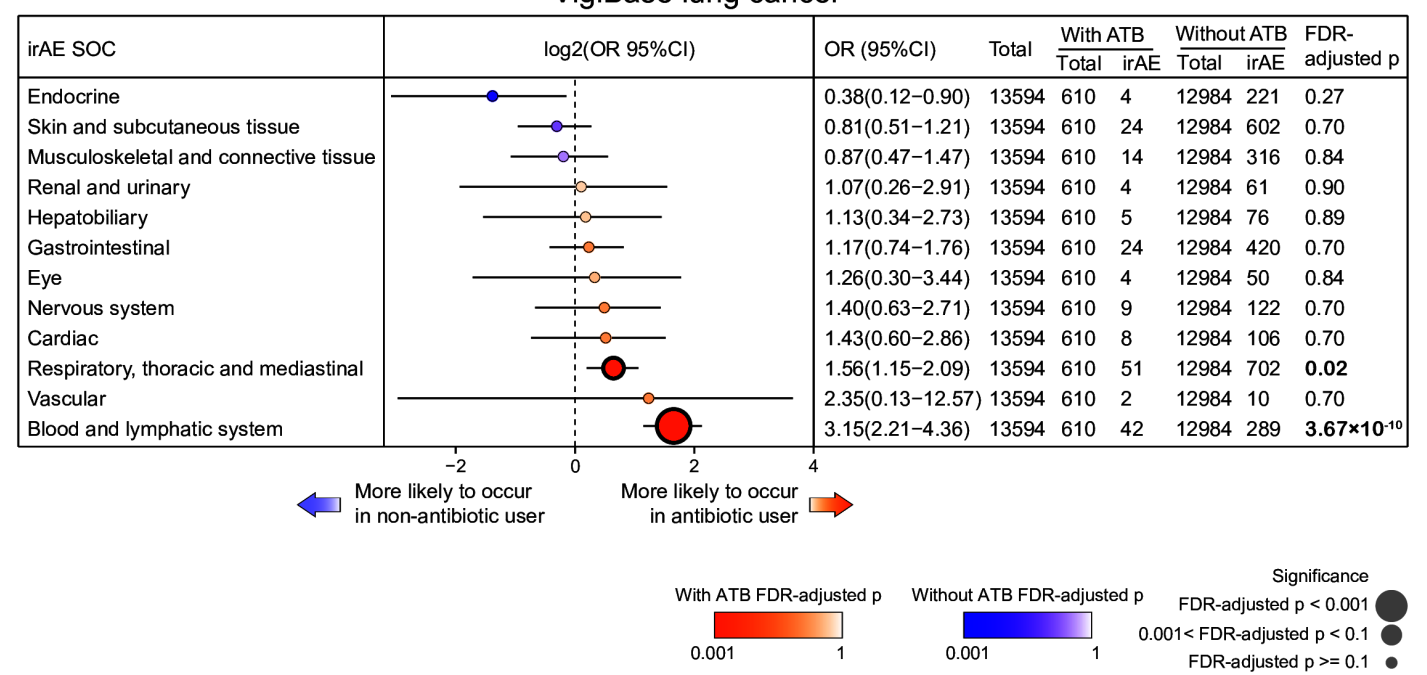

Figure 3 Multivariate logistic regression analysis of pharmacovigilance data for association between antibiotic use and irAEs from different organs. Analysis of irAEs in different organs/systems in antibiotic and non-antibiotic users among patients with lung cancer receiving anti-PD-1/PD-L1 from Food and Drug Administration Adverse Event Reporting System (FAERS) (A) and VigiBase (B). Dark orange indicates that immune-related adverse events (irAEs) are more likely to occur in antibiotic users; blue indicates that irAEs are more likely to occur in non-antibiotic users; shade of the dot indicates false discovery rate (FDR) adjusted-p value. Dot size from large to small, respectively, indicates FDR adjusted- $p<0.001,0.001<$ FDR adjusted-p $<0.1$, and FDR adjusted- $p \geq 0.1$. ATB, antibiotics.

\section{Molecular associations between microbial diversity and irAE risk}

Given the fact that use of antibiotics will decrease the microbial diversity, ${ }^{11} 42-44$ we hypothesized that decreased microbial diversity is associated with the increase of the irAE related factors. We took the advantage of multiomics data across 25 cancer types with more than 100 patient samples from TCGA (online supplemental figure S5) and obtained the microbial data $^{23}$ from a recent study to calculate microbial diversity based on the Inverse Simpson Index. ${ }^{45}$ We investigated the associations between microbial diversity and reported irAE-related factors and signatures: TMB, ${ }^{24}$ TCR diversity, ${ }^{25}$ neutrophils, ${ }^{26}$ eosinophils, ${ }^{26}$ CEACAM1, ${ }^{26}$ CD177, ${ }^{26}$ IFN alpha response, ${ }^{27}$ and TNF signature, ${ }^{27}$ Cytolytic activity, ${ }^{21}$ ADPGK $^{21}$ LCP1 $^{21}$ PD-1, ${ }^{21}$ and potential pathways ${ }^{28}$ related to $\mathrm{T}$ cell activation, neutrophil activation, eosinophils, and inflammation (online supplemental figure S4). We observed negative associations between microbial diversity and irAE related factors/pathways in multiple cancer types (online supplemental figure S4). Among these cancer types, two non-small cell lung cancer types, lung adenocarcinoma and lung squamous cell carcinoma, were negatively correlated with most irAE-related factors / pathways (figure 5A). Next, we performed correlation analysis between microbial diversity and gene expression (online supplemental figure S6), and those significantly correlated genes were enriched in neutrophil activation and $\mathrm{T}$ cell activation pathways (figure $5 \mathrm{~B}$ ). This result aligns well with the emergence of evidence of the involvement of neutrophil activation and/or T-cell activation in irAEs. Taken together, our observation suggested the potential mechanism that decreased microbial diversity caused by antibiotics use may increase the irAE risk through mediating the irAE-related factors (figure 5C).

\section{DISCUSSION}

To our knowledge, this study reports the most comprehensive assessment of the association between antibiotic 
A

FAERS lung cancer

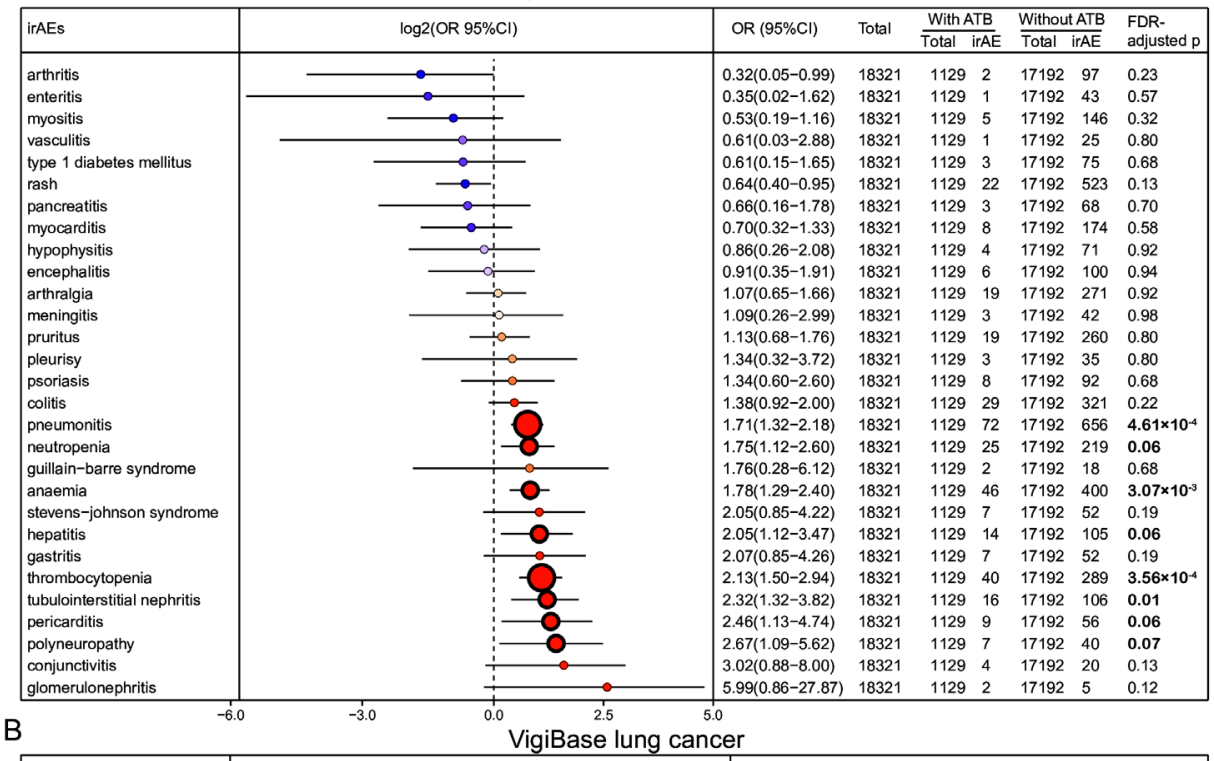

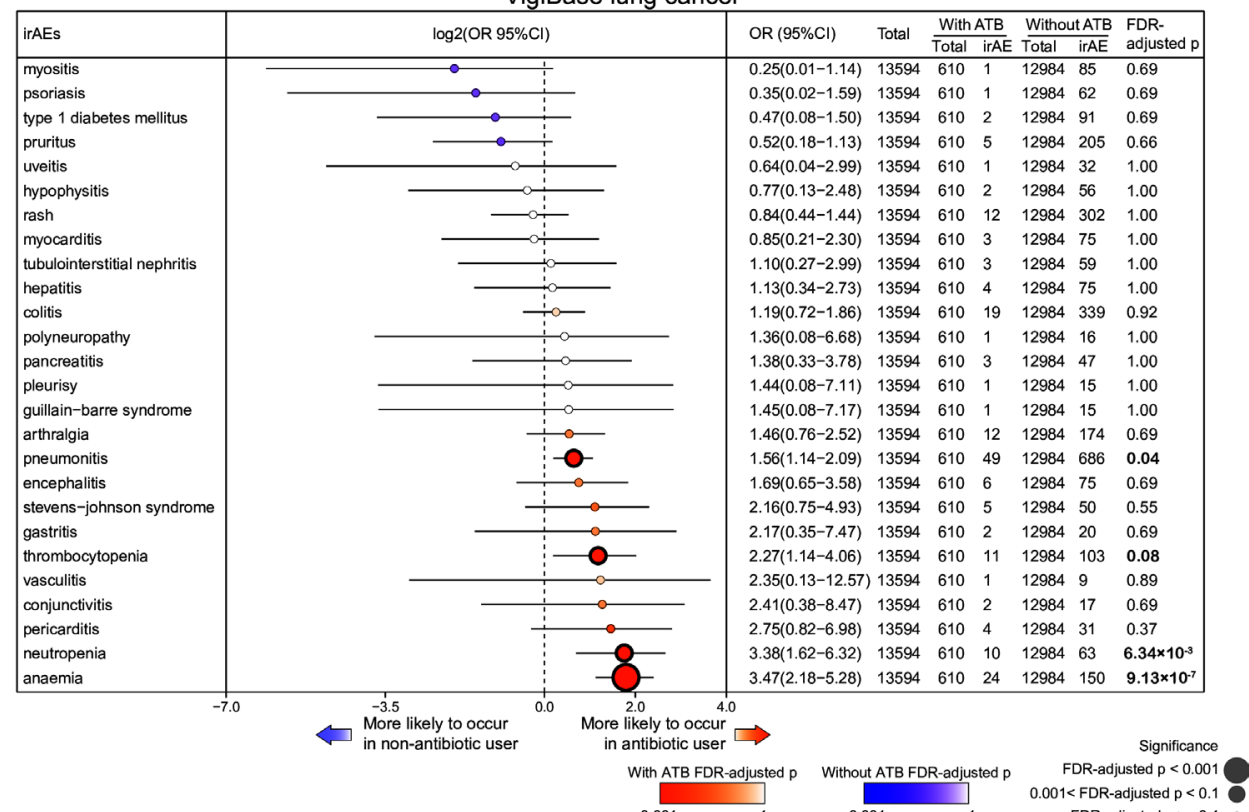

Multivariate logistic regression analysis of pharmacovigilance data for association between antibiotic use and individual immunerelated adverse events (irAEs). Analysis of individual irAE in antibiotic and non-antibiotic users among patients with lung cancer receiving anti-PD-1/PD-L1 from Food and Drug Administration Adverse Event Reporting System (FAERS) (A) and VigiBase (B). dark orange indicates that irAEs are more likely to occur in antibiotic users; blue indicates that irAEs are more likely to occur in nonantibiotic users; shade of the dot indicates false discovery rate (FDR) adjusted-p value. dot size from large to small respectively indicates FDR adjusted-p <0.001, 0.001<FDR adjusted-p <0.1, and FDR adjusted-p $\geq 0.1$. ATB, antibiotics.

use and irAE development in different organs during anti-PD-1/PD-L1 therapy across a wide spectrum of cancers by analyzing a retrospective cohort with a relatively large sample size and comprehensive analyses of two pharmacovigilance datasets. Analyzing real-world pharmacovigilance data provides an additional information source that not only increases the power of the analysis but includes standard of care information not available from clinical trials, ${ }^{4619244147}$ such as the inclusion of all irAEs, including those that may cause lethal outcomes (eg, myocarditis). We consistently observed increased irAE risk with antibiotic use among patients with lung cancer across different analysis. Further analysis of individual irAEs in patients with lung cancer from FAERS suggested that the risk of pneumonitis was higher among patients with lung cancer treated with antibiotics, which is also the first report to reveal the association between a wide range of specific irAEs and antibiotic treatment. Consistently, we also observed increased risk of pneumonitis among antibiotic users who were ICI patients with lung cancer from VigiBase, suggesting the robustness of our observation. Pneumonitis is the most common type of respiratory, thoracic and mediastinal irAE. Though the overall incidence of pneumonitis is low, ranging from $1.3 \%$ to $5 \%^{122}$ in clinical trials, it is potentially lifethreatening. ${ }^{4}$ Therefore, our study provided evidence 


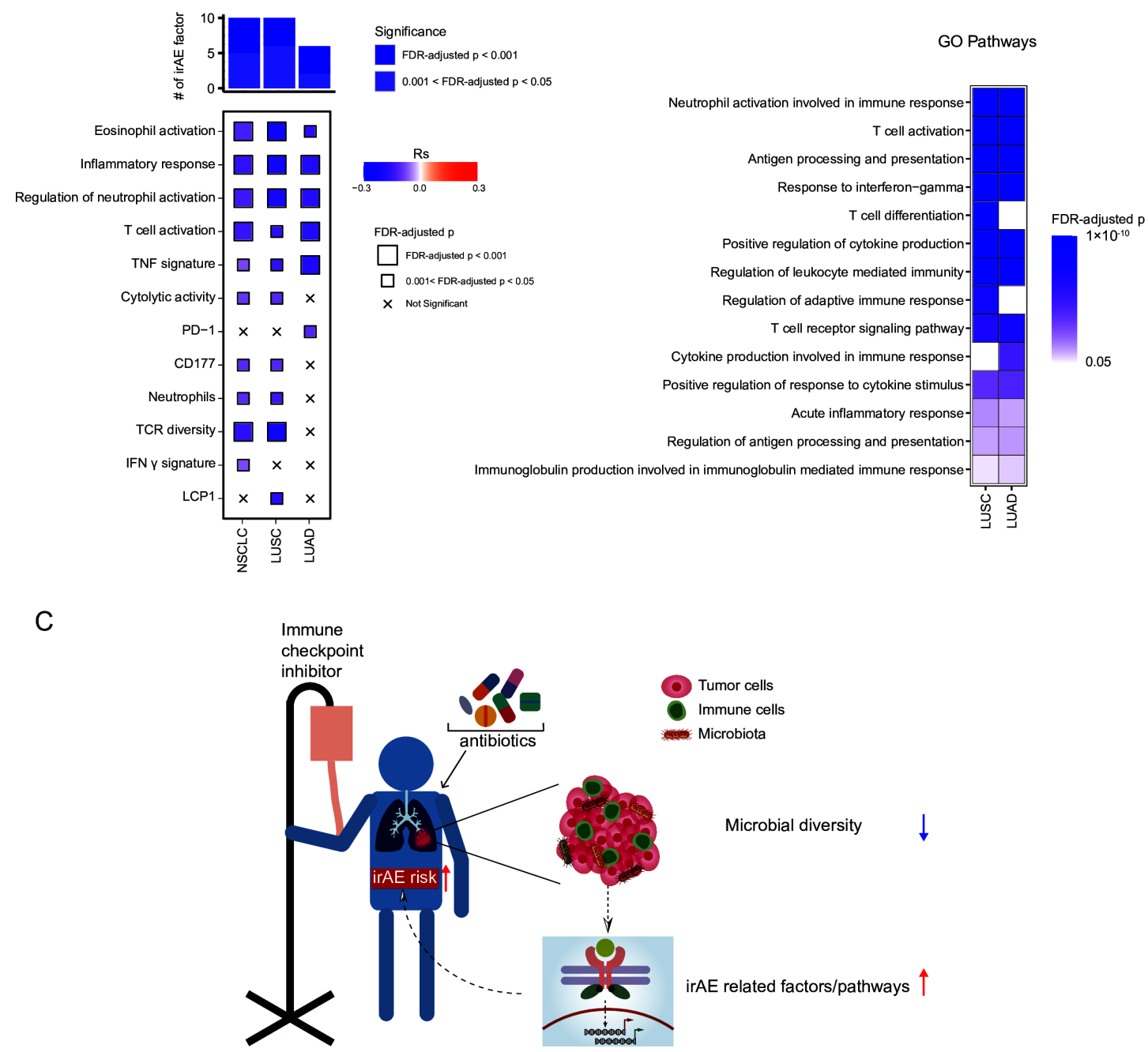

Figure 5 Putative molecular mechanisms of association between antibiotic usage and immune-related adverse event (irAE) risk. (A) Spearman correlation between microbial diversity and irAE related factors/pathways. Top bar plot, number of significantly associated irAE-related factors/pathways with positive (red) or negative correlation (blue). Inverse Simpson Index was employed to evaluate microbial diversity. Dot size from large to small, respectively, indicates false discovery rate (FDR) adjusted-p $<0.001$ and $0.001<F D R$ adjusted-p $<0.05$. Red arrow: increase; blue arrow: decrease. (B) pathway enrichment of genes significantly correlated with bacterial diversity in LUAD and LUSC. Spearman correlation was performed and absolute value of Spearman $R(R S) \geq 0.3$ and FDR adjusted- $p<0.05$ were considered as significant. Shade of the square indicated FDRadjusted p-value. (C) Graphical summary of potential mechanisms for antibiotics and irAEs. IFN, interferon; LCP1, lymphocyte cytosolic protein 1; LUAD, lung adenocarcinoma; LUSC, lung squamous cell carcinoma; NSCLC, non-small cell lung cancer by combination of LUAD and LUSC; TCR, T cell receptor; TNF, tumor necrosis factor.

that the administration of antibiotics should be carefully evaluated in patients with cancer treated by anti-PD-1/ PD-L1 to avoid potentially increasing irAE risk, at least for patients with lung cancer. Healthcare providers should carefully evaluate this risk before using antibiotics, and ICI patients should be carefully monitored if they have antibiotic administration.

Previous studies have reported an association between antibiotic use and higher risk of diarrhea and colitis in ICI patients, ${ }^{12} 13$ suggesting the potential impact of antibiotics on ICI-induced colitis through microbiomeimmune axis. In our study, we observed a trend for patients with lung cancer who used antibiotics to experience more colitis; however, our findings did not reach statistical significance, which was likely due to the limited sample size (figure 4A-B). Our observation of nongastrointestinal irAEs in patients with cancer suggests that antibiotics, which exert a direct impact on host cells, could directly influence irAE development via regulating the microbiome and immune environment of cancer cells or host tissue cells.

There are several limitations of our study. Currently, it is challenging to define an $\mathrm{AE}$ that is definitely immune related, mainly caused by substantial heterogeneity among irAEs, such as clinical manifestations, time of onset, and incidences. ${ }^{48}$ We, therefore, employed a prespecified list of irAE terms ${ }^{22}$ to determine patients with irAEs, including pneumonitis, colitis and hepatitis. This prespecified list is 
one of the most comprehensive and accurate irAE list, comprised of terms that were widely utilized in previous studies to study irAEs ${ }^{424}$ based on either pharmacovigilance data or clinical cohorts. Furthermore, due to the limited sample size or lacking the detailed information, some potential irAE-related risk factors, for example, $\mathrm{TMB},{ }^{24}$ are not considered in current study. Future studies that combine efforts from multiple centers will contribute to a deeper understanding of the association between irAEs and antibiotic use. In addition, real-world pharmacovigilance data are balanced by the quality and completeness, ${ }^{50}{ }^{51}$ such as the absence of irAE grading data, time and reason for antibiotic use, and duration of treatment, in FAERS and VigiBase, which prevent us from adjusting more confounding factors in the analysis, and we can only obtain potential risks through calculating OR based on these databases. For example, antibiotics could be administered for the treatment of possible irAEs or infections (eg, initial treatment for pneumonia vs pneumonitis), but limitations of the reporting system do not allow to distinguish between different causal relationship. We observed increased risks in both pre-ICI groups (eg, 3 months and 30 days) with antibiotics usage in our in-house patient cohort, suggesting that the association between irAEs and antibiotic usage is unlikely to be caused by concurrent antibiotics during irAE management.

TCGA has generated huge amount of multi-omics data from 33 different cancer types, and provides unique opportunities to comprehensively characterize molecular signatures and understand mechanisms of human cancers. ${ }^{2152-56}$ Though irAE information of these samples are lacking, it has been utilized to indirectly study irAEs. ${ }^{21} 2857$ Our analysis of TCGA multiomics data suggested the potential mechanism that decreased microbial diversity caused by antibiotics use may increase the irAE risk through mediating the irAE-related factors. Due to the complexity of the interactions between human microbiome and host cells ${ }^{58}$ and lacking irAE information for individual patients in these large-scale genomics consortia could lead to potential bias, further concerted efforts to systemically characterize cancer-associated microbiome as well as the multiomics data at patient level will be necessary to reveal the underlying mechanism of such associations.

\section{CONCLUSIONS}

Our comprehensive study, on a wide spectrum of patients with cancer treated by anti-PD-1/PD-L1, suggested that administration of antibiotics should be carefully evaluated to avoid potentially increasing irAE risk.

\footnotetext{
Author affiliations

${ }^{1}$ Clinical Research Institute, Shanghai Jiao Tong University School of Medicine, Shanghai, China

${ }^{2}$ Department of Biochemistry and Molecular Biology, The University of Texas Health Science Center at Houston McGovern Medical School, Houston, Texas, USA
}

${ }^{3}$ Early Clinical Trial Center, Office of National Drug Clinical Trial Institution, Hunan Cancer Hospital, The Affiliated Cancer Hospital of Xiangya School of Medicine, Central South University, Changsha, Hunan, China

${ }^{4}$ Center for Epigenetics and Disease Prevention, Institute of Biosciences and Technology, Texas A\&M University, Houston, Texas, USA

${ }^{5}$ Department of Radiation Oncology, University of Texas MD Anderson Cancer Center, Houston, Texas, USA

${ }^{6}$ Department of Bioinformatics and Computational Biology, University of Texas MD Anderson Cancer Center, Houston, Texas, USA

${ }^{7}$ Division of Hematology and Oncology, Mayo Clinic, Jacksonville, Florida, USA ${ }^{8}$ Department of Internal Medicine, Vanderbilt University Medical Center, Nashville, Tennessee, USA

${ }^{9}$ Department of Dermatology, Hunan Key Laboratory of Skin Cancer and Psoriasis, Hunan Engineering Research Center of Skin Health and Disease, Xiangya Clinical Research Center for Cancer Immunotherapy, Xiangya Hospital, Central South University, Changsha, Hunan, China

${ }^{10}$ Department of Translational Medical Sciences, College of Medicine, Texas A\&M University, Houston, TX, USA

Contributors LH conceived and supervised the project. YJ and LH designed and performed the research. LH is also responsible for the overall content as guarantor. YJ, YLou, YaL, ZZ, YC, YuL, YW and LD contributed to data analysis. YZ, XuC, KL, XiC and $\mathrm{HL}$ contributed to retrospective cohort generating and reviewing. YJ, YLou, SHL, $\mathrm{DBJ}, \mathrm{HL}, \mathrm{JW}$ and $\mathrm{LH}$ interpreted the results and wrote the manuscript.

Funding This work was supported by the Cancer Prevention \& Research Institute of Texas (RR150085 and RP190570 to CPRIT Scholar in Cancer Research, LH).

Competing interests DBJ is on the advisory boards or consults for BMS, Catalyst, lovance, Jansen, Merck, Mosaic ImmunoEngineering, Novartis, Oncosec, Pfizer, and Targovax, and receives research funding from BMS and Incyte. All other authors declare no competing interests.

\section{Patient consent for publication Not applicable.}

Ethics approval The study was conducted in accordance with ethical guidelines of US Common Rule and was approved by the Ethics Committee of Hunan Cancer Hospital, the Affiliated Cancer Hospital of Xiangya School of Medicine.

Provenance and peer review Not commissioned; externally peer reviewed.

Data availability statement Data are available on reasonable request. All associated data are available within the article, online supplemental files or available from the author on reasonable request.

Supplemental material This content has been supplied by the author(s). It has not been vetted by BMJ Publishing Group Limited (BMJ) and may not have been peer-reviewed. Any opinions or recommendations discussed are solely those of the author(s) and are not endorsed by BMJ. BMJ disclaims all liability and responsibility arising from any reliance placed on the content. Where the content includes any translated material, BMJ does not warrant the accuracy and reliability of the translations (including but not limited to local regulations, clinical guidelines, terminology, drug names and drug dosages), and is not responsible for any error and/or omissions arising from translation and adaptation or otherwise.

Open access This is an open access article distributed in accordance with the Creative Commons Attribution Non Commercial (CC BY-NC 4.0) license, which permits others to distribute, remix, adapt, build upon this work non-commercially, and license their derivative works on different terms, provided the original work is properly cited, appropriate credit is given, any changes made indicated, and the use is non-commercial. See http://creativecommons.org/licenses/by-nc/4.0/.

\section{ORCID iDs}

Steven H Lin http://orcid.org/0000-0003-4411-0634

Leng Han http://orcid.org/0000-0002-7380-2640

\section{REFERENCES}

1 Kennedy LB, Salama AKS. A review of cancer immunotherapy toxicity. CA Cancer J Clin 2020;70:86-104.

2 Ramos-Casals M, Brahmer JR, Callahan MK, et al. Immune-related adverse events of checkpoint inhibitors. Nat Rev Dis Primers 2020:6:1-21.

3 Esfahani K, Elkrief A, Calabrese C, et al. Moving towards personalized treatments of immune-related adverse events. Nat Rev Clin Oncol 2020;17:504-15. 
4 Wang DY, Salem J-E, Cohen JV, et al. Fatal toxic effects associated with immune checkpoint inhibitors: a systematic review and metaanalysis. JAMA Oncol 2018;4:1721-8.

5 Moslehi JJ, Salem J-E, Sosman JA, et al. Increased reporting of fatal immune checkpoint inhibitor-associated myocarditis. Lancet 2018;391:933.

6 Salem J-E, Manouchehri A, Moey M, et al. Cardiovascular toxicities associated with immune checkpoint inhibitors: an observational, retrospective, pharmacovigilance study. Lancet Oncol 2018;19:1579-89.

7 Brahmer JR, Lacchetti C, Schneider BJ, et al. Management of immune-related adverse events in patients treated with immune checkpoint inhibitor therapy: American Society of Clinical Oncology clinical practice guideline. J Clin Oncol 2018;36:1714-68.

8 Friedman CF, Proverbs-Singh TA, Postow MA. Treatment of the immune-related adverse effects of immune checkpoint inhibitors: a review. JAMA Oncol 2016;2:1346-53.

9 Postow MA, Sidlow R, Hellmann MD. Immune-related adverse events associated with immune checkpoint blockade. N Engl J Med 2018;378:158-68.

10 Derosa L, Hellmann MD, Spaziano M, et al. Negative association of antibiotics on clinical activity of immune checkpoint inhibitors in patients with advanced renal cell and non-small-cell lung cancer. Ann Oncol 2018;29:1437-44.

11 Pinato DJ, Howlett S, Ottaviani D, et al. Association of prior antibiotic treatment with survival and response to immune checkpoint inhibitor therapy in patients with cancer. JAMA Oncol 2019;5:1774-8.

12 Mohiuddin JJ, Chu B, Facciabene A, et al. Association of antibiotic exposure with survival and toxicity in patients with melanoma receiving immunotherapy. J Nat/ Cancer Inst 2021;113:162-70.

13 Abu-Sbeih H, Herrera LN, Tang T. Impact of antibiotic therapy on the development and response to treatment of immune checkpoint inhibitor-mediated diarrhea and colitis. J Immunother Cancer 2019;7:1-11.

14 Ramírez-Labrada AG, Isla D, Artal A, et al. The influence of lung microbiota on lung carcinogenesis, immunity, and immunotherapy. Trends Cancer 2020;6:86-97.

15 Xavier JB, Young VB, Skufca J, et al. The cancer microbiome: distinguishing direct and indirect effects requires a systemic view. Trends Cancer 2020;6:192-204.

16 Sepich-Poore GD, Zitvogel L, Straussman R, et al. The microbiome and human cancer. Science 2021;371. doi:10.1126/science.abc4552. [Epub ahead of print: 2603 2021]

17 Kaderbhai C, Richard C, Fumet JD, et al. Antibiotic use does not appear to influence response to nivolumab. Anticancer Res 2017;37:3195-200.

18 Oshima Y, Tanimoto T, Yuji K, et al. EGFR-TKI-associated interstitial pneumonitis in nivolumab-treated patients with non-small cell lung cancer. JAMA Oncol 2018;4:1112-5.

19 Zamami Y, Niimura T, Okada N, et al. Factors associated with immune checkpoint Inhibitor-Related myocarditis. JAMA Oncol 2019;5:1635-4.

20 Jing Y, Diao L, Han L. Adverse events associated with potential drugs for COVID-19: a case study from real-world data. Brief Bioinform 2021;22:1232-8.

21 Jing Y, Liu J, Ye Y, et al. Multi-Omics prediction of immune-related adverse events during checkpoint immunotherapy. Nat Commun 2020:11:1-7.

22 Martins F, Sofiya L, Sykiotis GP, et al. Adverse effects of immunecheckpoint inhibitors: epidemiology, management and surveillance. Nat Rev Clin Oncol 2019;16:563-80.

23 Poore GD, Kopylova E, Zhu Q, et al. Microbiome analyses of blood and tissues suggest cancer diagnostic approach. Nature 2020;579:567-74.

24 Bomze D, Hasan Ali O, Bate A, et al. Association between immunerelated adverse events during anti-PD-1 therapy and tumor mutational burden. JAMA Oncol 2019;5:1633-5.

25 Johnson DB, Balko JM, Compton ML, et al. Fulminant myocarditis with combination immune checkpoint blockade. N Engl J Med 2016;375:1749-55.

26 Manson G, Norwood J, Marabelle A, et al. Biomarkers associated with checkpoint inhibitors. Ann Oncol 2016;27:1199-206.

27 Head L, Gorden N, Van Gulick R, et al. Biomarkers to predict immune-related adverse events with checkpoint inhibitors. J Clin Oncol 2019;37:131.

28 Jing Y, Zhang Y, Wang J, et al. Association between sex and immune-related adverse events during immune checkpoint inhibitor therapy. J Natl Cancer Inst 2021;113:1396-404.

29 Thorsson V, Gibbs DL, Brown SD, et al. The immune landscape of cancer. Immunity 2018;48:e14:812-30.
30 Hellmann MD, Callahan MK, Awad MM, et al. Tumor mutational burden and efficacy of nivolumab monotherapy and in combination with ipilimumab in small-cell lung cancer. Cancer Cell 2018;33:853-61.

31 Perez-Ruiz E, Minute L, Otano I, et al. Prophylactic TNF blockade uncouples efficacy and toxicity in dual CTLA-4 and PD-1 immunotherapy. Nature 2019;569:428-32.

32 Ayers M, Lunceford J, Nebozhyn M, et al. IFN- $\gamma$-related mRNA profile predicts clinical response to PD-1 blockade. J Clin Invest 2017; 127:2930-40.

33 Hänzelmann S, Castelo R, Guinney J. GSVA: gene set variation analysis for microarray and RNA-Seq data. BMC Bioinformatics 2013;14:7.

34 Yu G, Wang L-G, Han Y, et al. ClusterProfiler: an R package for comparing biological themes among gene clusters. OMICS: $A \mathrm{~J}$ of Integrative Biology 2012;16:284-7.

35 Chalabi M, Cardona A, Nagarkar DR, et al. Efficacy of chemotherapy and atezolizumab in patients with non-small-cell lung cancer receiving antibiotics and proton pump inhibitors: pooled post hoc analyses of the oak and poplar trials. Ann Oncol 2020;31:525-31.

36 Cortellini A, Tucci M, Adamo V, et al. Integrated analysis of concomitant medications and oncological outcomes from PD-1/PDL1 checkpoint inhibitors in clinical practice. J Immunother Cancer 2020;8.

37 Cortellini A, Ricciuti B, Facchinetti F, et al. Antibiotic-exposed patients with non-small-cell lung cancer preserve efficacy outcomes following first-line chemo-immunotherapy. Ann Oncol 2021;32:1391-9.

38 Cortellini A, Di Maio M, Nigro O, et al. Differential influence of antibiotic therapy and other medications on oncological outcomes of patients with non-small cell lung cancer treated with first-line pembrolizumab versus cytotoxic chemotherapy. J Immunother Cancer 2021;9:e002421.

39 Das S, Johnson DB. Immune-Related adverse events and anti-tumor efficacy of immune checkpoint inhibitors. J Immunother Cancer 2019;7:1-11.

40 June $\mathrm{CH}$, Warshauer JT, Bluestone JA. Is autoimmunity the Achilles' heel of cancer immunotherapy? Nat Med 2017;23:540-7.

41 Suchard MA, Schuemie MJ, Krumholz HM, et al. Comprehensive comparative effectiveness and safety of first-line antihypertensive drug classes: a systematic, multinational, large-scale analysis. Lancet 2019;394:1816-26.

42 Weber D, Hiergeist A, Weber M, et al. Detrimental effect of broadspectrum antibiotics on intestinal microbiome diversity in patients after allogeneic stem cell transplantation: lack of commensal sparing antibiotics. Clin Infect Dis 2019;68:1303-10.

43 Elkrief A, Derosa L, Kroemer G, et al. The negative impact of antibiotics on outcomes in cancer patients treated with immunotherapy: a new independent prognostic factor? Ann Oncol 2019;30:1572-9.

44 Gopalakrishnan V, Spencer CN, Nezi L, et al. Gut microbiome modulates response to anti-PD-1 immunotherapy in melanoma patients. Science 2018;359:97-103.

45 Schluter J, Peled JU, Taylor BP, et al. The gut microbiota is associated with immune cell dynamics in humans. Nature 2020;588:303-7.

46 Wang $\mathrm{Y}$, Wiesnoski DH, Helmink BA, et al. Fecal microbiota transplantation for refractory immune checkpoint inhibitor-associated colitis. Nat Med 2018;24:1804-8.

47 Nabhan C, Klink A, Prasad V. Real-world evidence - what does it really mean? JAMA Oncol 2019;5:781-3.

48 Dougan M, Luoma AM, Dougan SK, et al. Understanding and treating the inflammatory adverse events of cancer immunotherapy. Cell 2021;184:1575-88.

49 Dolladille C, Ederhy S, Sassier M, et al. Immune checkpoint inhibitor rechallenge after immune-related adverse events in patients with cancer. JAMA Oncol 2020;6:865-71.

50 Verzoni E, Cartenì G, Cortesi E, et al. Real-World efficacy and safety of nivolumab in previously-treated metastatic renal cell carcinoma, and association between immune-related adverse events and survival: the Italian expanded access program. $J$ Immunother Cancer 2019;7:1-9.

51 Johnson DB, Manouchehri A, Haugh AM, et al. Neurologic toxicity associated with immune checkpoint inhibitors: a pharmacovigilance study. J Immunother Cancer 2019;7:1-9.

52 Cancer Genome Atlas Research Network, Weinstein JN, Collisson $\mathrm{EA}$, et al. The cancer genome atlas pan-cancer analysis project. Nat Genet 2013;45:1113-20.

53 Campbell PJ, Getz G, Korbel JO, et al. Pan-cancer analysis of whole genomes. Nature 2020;578:82-93. 
54 Yuan Y, Liu L, Chen H, et al. Comprehensive characterization of molecular differences in cancer between male and female patients. Cancer Cell 2016;29:711-22.

55 Ye Y, Jing Y, Li L, et al. Sex-associated molecular differences for cancer immunotherapy. Nat Commun 2020;11:11.

$56 \mathrm{Ye} \mathrm{Y}, \mathrm{Hu} \mathrm{Q}$, Chen $\mathrm{H}$, et al. Characterization of hypoxia-associated molecular features to aid hypoxia-targeted therapy. Nat Metab 2019;1:431-44.
57 Berner F, Bomze D, Diem S, et al. Association of checkpoint inhibitor-induced toxic effects with shared cancer and tissue antigens in non-small cell lung cancer. JAMA Oncol 2019;5:1043-7.

58 Nejman D, Livyatan I, Fuks G, et al. The human tumor microbiome is composed of tumor type-specific intracellular bacteria. Science 2020;368:973-80. 